\title{
Separability and Independence of Congestion and Reliability: Theory and Simulations
}

\author{
Seth Blumsack, Member, IEEE, Marija Ilić, Fellow, IEEE and Lester B. Lave
}

\begin{abstract}
One policy aim under electric-industry restructuring has been to replace the centralized utility planning process (integrated resource planning) with regional planning for network investments, and decentralized decision-making for merchant investments. Through specific policies such as encouraging market-based merchant transmission and the proposed establishment of National Interest Transmission Corridors under the Energy Policy Act of 2005, congestion and reliability have been treated as separable and independent system attributes. This paper brings together separate pieces of research in [1] - [3] to explore the proposition that transmission investments can be divided into those which relieve congestion and those which enhance system reliability. Using the DC power flow model with linear ATC, we derive some explicit necessary and sufficient analytical conditions under which the separability and independence assumptions hold. These include (i) the network is series-parallel; (ii) demand is completely priceinelastic; (iii) all customers value reliability identically; and (iv) the grid operator does not discriminate among customers when forced to physically ration consumption. Simulations using the IEEE 118-bus test system are presented which demonstrate the close dependence of reliability and congestion, except at very low levels of demand.
\end{abstract}

Index Terms-Reliability, congestion, merchant transmission, transmission investment, available transfer capacity, Braess Paradox, Wheatstone network.

\section{NOMENCLATURE}

$N L=$ Number of lines in the network

$N B=$ Number of buses in the network

$S_{i j}=$ Transmission line connecting buses $i$ and $j$

$B_{i j}=$ Susceptance of the link connecting buses $i$ and $j$

$\theta_{i}=$ Phase angle at the $i$ th bus

$P_{i}=$ Net real power injection at the $i$ th bus; positive for net generation and negative for net withdrawal

This work was funded by a grant to the Carnegie Mellon Electricity Industry Center from EPRI and the Alfred P. Sloan foundation, and by the Tennessee Valley Authority. Any opinions and errors are those of the authors and should not be ascribed to the grantors

Seth Blumsack is a postdoctoral researcher at the Tepper School of Business, Carnegie Mellon University, Pittsburgh PA 15213 (email: blumsack@cmu.edu).

Marija Ilić is Professor of Electrical and Computer Engineering and Enginering and Public Policy, Carnegie Mellon University, Pittsburgh PA 15213 (email: milic@ece.cmu.edu).

Lester B. Lave is the Harry and James Higgins Professor of Economics and Finance, Tepper School of Business, Carnegie Mellon University, Pittsburgh PA and Co-Director of the Carnegie Mellon Electricity Industry Center (email: lave@cmu.edu).
$P_{L i}=$ Real power demand at the $i$ th bus

$P_{G i}=$ Real power demand at the $i$ th bus

$\rho_{l i}=$ Power transfer distribution factor along line $l$ with

respect to a network resource at bus $i$.

$\delta_{i j}=$ Phase angle difference between buses $i$ and $j$

$F_{i j}=$ Real power flow between buses $i$ and $j$

$\pi_{i}=$ Nodal price at bus $i$

$\mu_{i j}=$ Shadow price of transmission between buses $i$ and $j$

$C_{i}=$ Total cost function at the $i$ th bus.

$A T C_{i}=$ Available transfer capacity into bus $i$.

$\mathbf{B}=(N B \times N B)$ system susceptance matrix

$\mathbf{B}^{\text {diag }}=(N L \times N L)$ diagonal matrix of line susceptances

$\mathbf{N}=(N B \times N B)$ node-node adjacency matrix

$\mathbf{A}=(N B \times N L)$ system node-line adjacency matrix

$\mathbf{P}=(N B \times 1)$ vector of bus injections

$\mathbf{F}=(N L \times 1)$ vector of line flows

$\theta=(N B \times 1)$ vector of bus angles

$\delta=(N L \times 1)$ vector of bus angle differences

$\rho=(N L \times N B)$ matrix of power transfer distribution factors

\section{INTRODUCTION}

$\mathrm{N}$ o matter what metrics are used, the transmission grid in the U.S. has become increasingly stressed since the onset of restructuring in the electric power sector. Spot energy markets run by Regional Transmission Organizations (RTO) have encouraged more long-distance transactions; the result has been more intense utilization of certain segments of the transmission grid. In systems that have not undergone restructuring, congestion is managed using transmission loading relief (TLR) procedures [4]. The incidence of TLR actions has increased by multiple orders of magnitude since restructuring, as shown in Figure 1. In restructured systems, congestion is managed using a combination of TLRs and market-based congestion pricing. Figure 2 shows the increase in total and average congestion charges in PJM. Even apart from the August, 2003 blackout, the frequency of large-scale disturbances appears to have increased since the opening of regional power markets in 1998 [5].

Transmission policy under restructuring originally tried to encourage the development of a merchant transmission sector, with congestion prices in RTO markets serving both to signal where new transmission was needed, and to compensate investors. Point-to-point financial transmission rights (FTR) were originally suggested by [6], and were found to have appealing economic efficiency properties by [7]. Tradeable 
flowgate rights, based on the analysis in [8] arose as an alternative structure to FTRs; the analysis of [9] suggested that flowgate rights could be more efficient than FTRs when generators had market power. More general criticism of the merchant transmission model can be found in [10] , [11], [1].

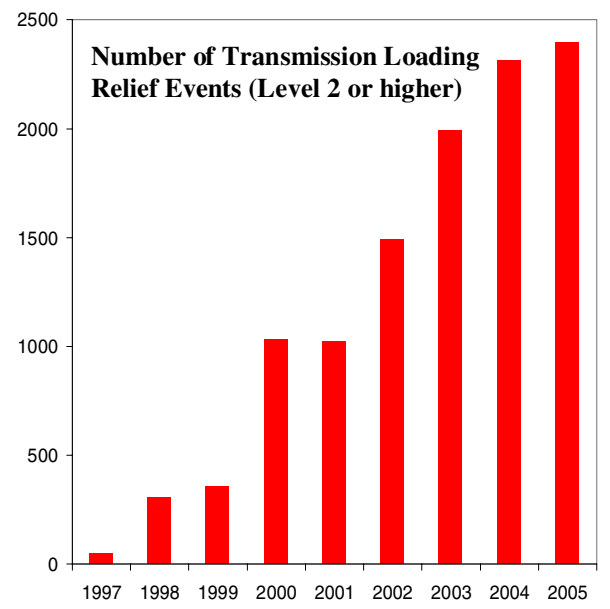

Figure 1: Transmission loading relief actions of Level 2 and higher. Source: NERC.

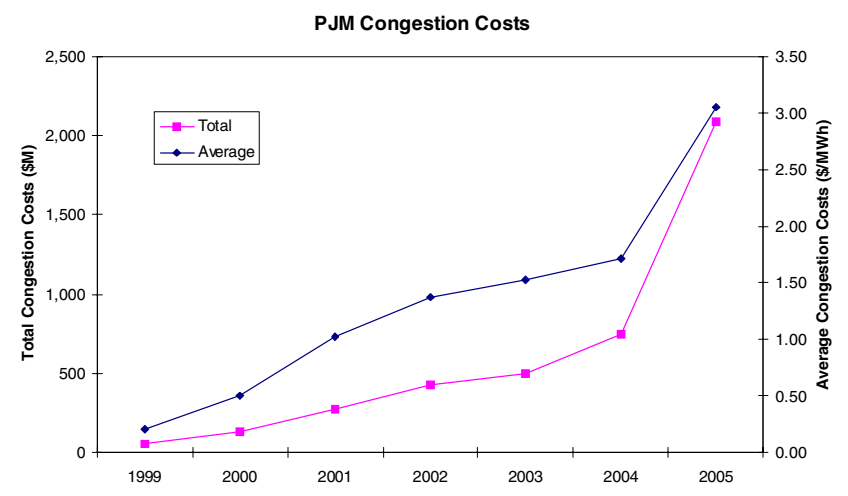

Figure 2: Total and average congestion costs in PJM. Source: PJM State of the Market Reports.

Success with the merchant transmission model in the U.S. has been limited. Non-utility companies have made transmission investments, but they have been compensated with some form of contract unrelated to market prices [12]. Still, language supporting market-based merchant transmission based on FTRs exists in the tariffs of the regional transmission operators such as PJM, New York ISO, and ISO New England.

The "pure" version of market-based transmission investment, as in [7], has given way to some more highlyadministered alternatives. Section 1221 of the U.S. Energy Policy Act of 2005 would allow the Department of Energy to designate National Interest Transmission Corridors (NITC) based on the results of a National Transmission Congestion Study [13]. FERC is then given authorization to intervene and expedite siting in the NITCs if the usual state-level regulatory processes are deemed to be moving too slowly. A slightly less pure form of market-based merchant transmission has been proposed in [14] and [15] as "participant funding." This scheme would permit socialization and central planning of transmission investments deemed necessary for reliability, whereas defined beneficiaries would pay for transmission investments needed for economic reasons.

Underlying the market-based merchant transmission model and its alternatives is an assumption that transmission projects can be cleanly separated into those that relieve congestion and those that enhance reliability. This distinction is clearly the most explicit in the participant funding models. Paul Joskow [16] argues that the distinction between investments for economics and those for reliability amounts to a meaningless dichotomy, since most transmission investments in the U.S. have been made by regulated utilities on the basis of reliability criteria. We agree with Joskow, but we argue a stronger point: that the distinction between congestion and reliability is often simply wrong. Lines that cause congestion in the network may be justified on reliability grounds, and vice versa.

\section{A STYLIZED EXAMPLE}

The interaction between congestion and reliability can be illustrated using the simple four-bus test network shown in Figure 3. This test system is known as the Wheatstone network, and the link connecting buses 2 and 3 is known as the Wheatstone bridge. Bus 1 is assumed to have an inexpensive generator with $P_{G 1}^{\max }=100 \mathrm{MW}$, while bus 4 has an expensive generator and a load with a constant per-period real power demand of $P_{L 1}=100 \mathrm{MW}$. Buses 2 and 3 are tiepoints containing neither generation nor load. In the context of the DC power flow model, the susceptances of lines $S_{12}$ and $S_{34}$ are assumed to be equal, and the susceptances of lines $S_{13}$ and $S_{24}$ are assumed to be equal. The two "upstream" lines $\left(S_{12}\right.$ and $\left.S_{13}\right)$ have a rated limit of $55 \mathrm{MW}$ each, and the two "downstream" lines $\left(S_{24}\right.$ and $\left.S_{34}\right)$ have a rated limit of 100 MW each.

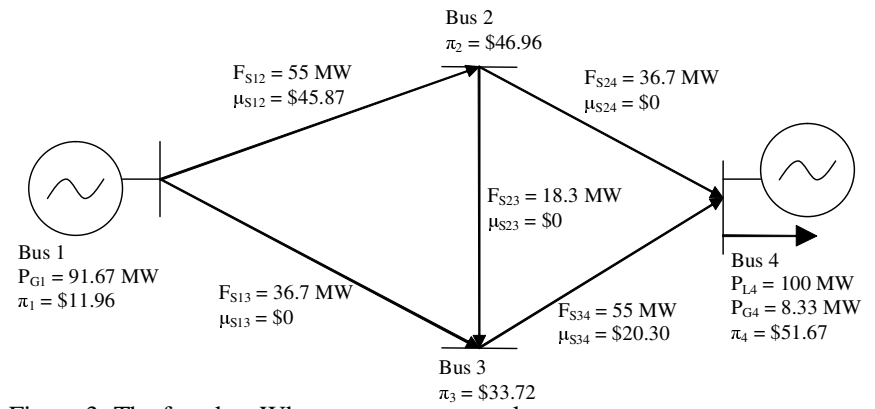

Figure 3: The four-bus Wheatstone test network.

The cost curves of the two generators are assumed to be quadratic with the following parameterization:

$$
\begin{gathered}
C\left(P_{G I}\right)=200+10.3 P_{G 1}+0.009 P_{G I}^{2} \\
C\left(P_{G 4}\right)=300+50 P_{G 4}+0.1 P_{G 4}^{2} .
\end{gathered}
$$


Without the Wheatstone bridge (and ignoring losses and reactive power), the inexpensive generator at bus 1 will produce $100 \mathrm{MW}$ and serve the entire load. Due to the symmetry in the network, $50 \mathrm{MW}$ will flow along each of the two paths from bus 1 to bus 4 . The network will have identical locational marginal prices (LMP) of \$12.11/MWperiod at each of the four buses; the total system cost of serving the load is $\$ 1,620$ per period.

Once the Wheatstone bridge is added to the network, the pattern of flows shifts, causing congestion on lines $S_{12}$ and $S_{34}$ in the network. ${ }^{1}$ The generator at bus 1 produces only 91.67 MW of real power, while the remainder must be made up by the expensive generator at bus 4 . The presence of congestion alters the LMPs at all four buses in the system; in particular, the LMP at the load bus increases to $\$ 51.67 / \mathrm{MW}$-period. The total system cost of serving the load rises to $\$ 1,945$ per period. The phenomenon that congestion is caused or worsened by adding links to a network is known as the Braess Paradox, and was first observed in automotive highway networks [17], [18]. A detailed discussion of the conditions under which the Braess Paradox will arise for more general electric networks can be found in [1 Ch. 3] and [19].

The behavior of the network in Figure 3 has the following implications for congestion management and transmission planning:

1. The network in Figure 3 represents a system where an investor could profit from building a line that reduced network capacity [1 Ch. 4], even if FTRs are allocated according to the "feasibility allocation rule." [6], [7].

2. Relieving congestion through network investments requires upgrades across the system. Upgrading just one of the two congested lines will not alter flows through the network or reduce the total cost of serving the load.

3. LMPs can provide a signal that congestion exists somewhere in the network, but cannot always suggest the least-cost strategy for relieving congestion. A system planner looking only at LMPs would upgrade lines $S_{12}$ or $S_{34}$. But congestion in the network could also be relieved by removing the Wheatstone bridge (or at least opening the circuit), perhaps at a lower cost. Thus, relieving congestion is a more complex problem than just upgrading the most congested line or lines.

The Wheatstone bridge harms the network by causing congestion but might be justified on reliability grounds. Suppose an outage were to occur on either of the two "downstream" lines $S_{24}$ or $S_{34}$. Without the Wheatstone bridge in the system, the loss of one of these downstream lines would effectively remove one of the electrical paths between buses 1 and 4 . Thus, only 55 MW could be

\footnotetext{
${ }^{1}$ These lines correspond to the high-susceptance lines in the network.
}

generated at bus 1 . If the generator at bus 4 has $P_{G 4}^{\max }<45$ MW, then unserved energy will result at bus 4 .

\section{Decomposition of Single ANd Multiple ElEment CHANGES IN NETWORK TOPOLOGY}

Through the example in Section III, we have implicitly defined the congestion effect of a given line as being related to a single-element change in the network topology (namely, the addition of the Wheatstone bridge to the network). Similarly, we have defined the reliability effect as being associated with a multiple-element change in the network topology (the addition of the Wheatstone bridge and the loss of one of the transmission lines on the boundary). We will explicitly define congestion and reliability metrics in Section $\mathrm{V}$, but in this section we will review the analysis of [2], which use the method of [20] and [21] to model single-element changes in the network topology. They extend this method to multiple-element topological changes, and demonstrate that a multiple-element topology change is mathematically equivalent to a sequence of single-element topology changes.

\section{A. Decomposition of Single-Element Topology Changes}

We first review the case of a single-element topology change; the goal is to decompose the effect of a change in network topology so as to be able to write:

$$
\mathbf{F}^{\text {new }}=\mathbf{F}^{\text {old }}+\Delta \mathbf{F}
$$

where $\Delta \mathbf{F}$ represents the adjustment factor due to a single change in the network topology. We start with the DC model for net injections:

$$
\mathbf{P}=\mathbf{B} \boldsymbol{\theta} .
$$

We model a change in the network topology as an adjustment to the susceptance matrix $\mathbf{B}$. The adjustment takes the form $\Delta \mathbf{B}_{k}=\mathbf{A} \Delta \mathbf{B}_{k}^{\text {diag }} \mathbf{A}^{\prime}$, where $\Delta \mathbf{B}_{k}^{\text {diag }}$ is a diagonal matrix whose entries are all equal to zero except the $k, k$ th entry, which is equal to $\Delta B_{k}$ (thus, $\Delta B_{k}$ represents a singleelement change in the network topology affecting line $k$ ). In the case of a line outage, we will have $\Delta B_{k}=-B_{k}$, and the dimensionality of $\Delta \mathbf{B}_{k}^{\text {diag }}$ will be $(N L \times N L)$. In the case of a network addition, we will have $\Delta B_{k}=+B_{k}$. The dimensionality of $\Delta \mathbf{B}_{k}^{\text {diag }}$ will be $(N L+1 \times N L+1)$, and the dimensionality of $\mathbf{A}$ will be $(N B \times N L+1)$, to account for the new line in the system. ${ }^{2}$

Following the change to the network topology, the DC equations can be written as:

\footnotetext{
${ }^{2}$ We could also model upgrades to the existing topology in a similar fashion. A line upgrade (not a line addition) would be represented by $\Delta B_{k}=+B_{k}$, with $+B_{k}$ being the magnitude of the upgrade. The dimensionality of $\Delta \mathbf{B}^{\text {diag }}$ would continue to be $(N L \times N L)$.
} 


$$
\mathbf{P}=\left(\mathbf{B}+\Delta \mathbf{B}_{k}\right) \boldsymbol{\theta} .
$$

Since $\Delta \mathbf{B}_{k}$ has rank one, we can write $\Delta \mathbf{B}_{k}=\Delta B_{k} \mathbf{A}_{k} \mathbf{A}_{k}^{\prime}$, where $\mathbf{A}_{k}$ is the $k$ th column of the incidence matrix $\mathbf{A}$.

To solve for $\boldsymbol{\theta}$, we use the Sherwood-Morrison-Woodbury matrix inversion lemma:

$$
\begin{aligned}
& \boldsymbol{\theta}^{\text {new }}=\left(\mathbf{B}+\Delta \mathbf{B}_{k}\right)^{-1} \mathbf{P} \\
& \quad=\left(\mathbf{B}^{-1}-\mathbf{B}^{-1} \mathbf{A}_{k}\left(1+\Delta B_{k} \mathbf{A}_{k}^{\prime} \mathbf{B}^{-1} \mathbf{A}_{k}\right)^{-1} B_{k} \mathbf{A}_{k}^{\prime} \mathbf{B}^{-1}\right) \mathbf{P} \\
& \Rightarrow \boldsymbol{\theta}^{\text {new }}=\boldsymbol{\theta}^{\text {old }}-\frac{\Delta B_{k} \mathbf{B}^{-1} \mathbf{A}_{k} \mathbf{A}_{k}^{\prime} \mathbf{\theta}^{\text {old }}}{1+\Delta B_{k} \mathbf{A}_{k}^{\prime} \mathbf{B}^{-1} \mathbf{A}_{k}},
\end{aligned}
$$

where we note that $1+B_{k} \mathbf{A}_{k}^{\prime} \mathbf{B}^{-1} \mathbf{A}_{k}$ is a scalar. We can insert (5) into the DC flow equations to yield:

$$
F_{l}^{\text {new }}=\left\{\begin{array}{l}
F_{l}^{\text {old }}+b_{k}^{-1} \mathbf{A}_{l}^{\prime} \mathbf{B}^{-\mathbf{1}} \mathbf{A}_{k} B_{l} \delta_{k}^{\text {old }}, \quad l \neq k \\
\left(F_{l}^{\text {old }}-\Delta B_{l} \delta_{l}^{\text {old }}\right)\left(1-b_{l}^{-1} \mathbf{A}_{l}^{\prime} \mathbf{B}^{-\mathbf{1}} \mathbf{A}_{l}\right), \quad l=k,
\end{array}\right.
$$

where $b_{k}=\left(\Delta B_{k}^{-1}+\mathbf{A}_{k}{ }^{\prime} \mathbf{B}^{-1} \mathbf{A}_{k}\right), b_{l}=\left(\Delta B_{l}^{-1}+\mathbf{A}_{l}{ }^{\prime} \mathbf{B}^{-1} \mathbf{A}_{l}\right)$.

\section{B. Decomposition of Multiple-Element Topology Changes}

We now show that (6) can be extended to the case of a change in the network topology affecting $n$ distinct network elements. Such a multi-element topology change can be modeled as a series of single-element topology changes (that is, some version of superposition holds). The network adjustment is again modeled as an adjustment of the form $\Delta \mathbf{B}=\mathbf{A} \Delta \mathbf{B}^{\text {diag }} \mathbf{A}^{\prime}$, except we allow $\Delta \mathbf{B}^{\text {diag }}$ to have multiple non-zero entries $\Delta B_{k k}, k=\{1, \ldots, n\}$ on the main diagonal (the off-diagonal entries are still assumed to be zero). If we assume that $n l$ of these network changes represent line outages, and $n 2$ of these network changes represent new lines (so we have $n 1+n 2=n$ ), then the dimensionality of $\Delta \mathbf{B}^{\text {diag }}$ will be $(N L+n 2 \times N L+n 2)$. We can write a general $n$ element topology change as:

$$
\Delta \mathbf{B}^{\text {diag }}=\Delta \mathbf{B}_{1}^{\text {diag }}+\Delta \mathbf{B}_{2}^{\text {diag }}+\cdots+\Delta \mathbf{B}_{n}^{\text {diag }} .
$$

We note that the rank of $\Delta \mathbf{B}^{\text {diag }}$ is equal to $n$, the number of distinct adjustments to the network topology. Thus, the rank of $\Delta \mathbf{B}=\mathbf{A} \Delta \mathbf{B}^{\text {diag }} \mathbf{A}^{\prime}$ is also equal to $n$.

Under a multi-element topology change (5) becomes:

$$
\begin{aligned}
\boldsymbol{\theta}= & \left(\mathbf{B}+\mathbf{A} \Delta \mathbf{B}^{\text {diag }} \mathbf{A}\right)^{-1} \mathbf{P} \\
& =\left(\mathbf{B}^{-1}-\mathbf{B}^{-1} \mathbf{A}\left(\mathbf{I}+\Delta \mathbf{B}^{\text {diag }} \mathbf{A}^{\prime} \mathbf{B}^{-1} \mathbf{A}\right)^{-1} \Delta \mathbf{B}^{\text {diag }} \mathbf{A}^{\prime} \mathbf{B}^{-1}\right) \mathbf{P},
\end{aligned}
$$

where $\mathbf{I}$ is the $(N L \times N L)$ identity matrix. The change in the bus angles is:

$$
\Delta \boldsymbol{\theta}=-\mathbf{B}^{-1} \mathbf{A}\left(\mathbf{I}+\Delta \mathbf{B}^{\text {diag }} \mathbf{A}^{\prime} \mathbf{B}^{-1} \mathbf{A}\right)^{-1} \Delta \mathbf{B}^{\text {diag }} \mathbf{A}^{\prime} \boldsymbol{\theta}^{\text {old }} .
$$

If we let $\mathbf{R}=\mathbf{B}^{-1} \mathbf{A}$, and $\mathbf{C}=\left(\mathbf{I}+\Delta \mathbf{B}^{\text {diag }} \mathbf{A}^{\prime} \mathbf{B}^{-1} \mathbf{A}\right)$, we get:

$$
\Delta \boldsymbol{\theta}=-\mathbf{R C}^{-1} \mathbf{A}^{\prime} \boldsymbol{\theta}^{\text {old }}
$$

If we further define $\boldsymbol{\Gamma}=\mathbf{C}^{-1} \mathbf{A}^{\prime} \boldsymbol{\theta}^{\text {old }}$, then we can write:

$$
\Delta \boldsymbol{\theta}=-\mathbf{R} \boldsymbol{\Gamma}=-\gamma_{1} \mathbf{r}_{1}-\gamma_{2} \mathbf{r}_{2}-\cdots-\gamma_{N L} \mathbf{r}_{N L},
$$

where $\gamma_{i}$ is the $i$ th element of $\boldsymbol{\Gamma}$, and $\mathbf{r}_{i}$ is the $i$ th column of $\mathbf{R}$.

The general form for (6) is thus:

$$
\begin{aligned}
\mathbf{F}^{\text {new }} & =\left(\mathbf{B}^{\text {diag }}+\Delta \mathbf{B}^{\text {diag }}\right) \mathbf{A}^{\prime}\left(\boldsymbol{\theta}^{\text {old }}-\mathbf{R} \boldsymbol{\Gamma}\right) \\
& =\mathbf{F}^{\text {old }}-\left(\Delta \mathbf{B}^{\text {diag }} \boldsymbol{\delta}^{\text {old }}-\sum_{i=1}^{n} \gamma_{i}\left(\mathbf{B}^{\text {diag }}+\Delta \mathbf{B}^{\text {diag }}\right) \mathbf{A}^{\prime} \mathbf{r}_{i}\right)
\end{aligned}
$$

\section{Variation of Distribution Factors with Topological Changes}

Ross Baldick [22] has demonstrated that even in the full AC power flow model, the distribution factors are approximately constant with network loadings. This may not be true when the network topology changes. Here we derive an expression showing how the matrix of power transfer distribution factors (PTDF) changes following alterations to the network topology. We begin with the definition of the PTDF matrix:

$$
\mathbf{\rho}=\mathbf{H B}^{-1},
$$

Where $\mathbf{H}$ is a $(N L \times N B)$ matrix defined by $\mathbf{H}=\mathbf{B}^{\text {diag }} \mathbf{A}^{\prime}$. Following a change to the network topology represented by $\Delta \mathbf{B}^{\text {diag }}$, we have:

$$
\mathbf{\rho}^{\text {new }}=\left(\mathbf{B}^{\text {diag }}+\Delta \mathbf{B}^{\text {diag }}\right) \mathbf{A}^{\prime}\left[\mathbf{A}\left(\mathbf{B}^{\text {diag }}+\Delta \mathbf{B}^{\text {diag }}\right) \mathbf{A}^{\prime}\right]^{-1} .
$$

Distributing terms in the inverse matrix in (20), we get:

$$
\begin{gathered}
\mathbf{\rho}^{\text {new }}=\left(\mathbf{B}^{\text {diag }} \mathbf{A}^{\prime} \mathbf{B}^{-1}\right)+\mathbf{B}^{\text {diag }} \mathbf{A}^{\prime} \Delta \mathbf{B}^{-1}+\Delta \mathbf{B}^{\text {diag }} \mathbf{A}^{\prime} \mathbf{B}^{-1} \\
+\Delta \mathbf{B}^{\text {diag }} \mathbf{A}^{\prime} \Delta \mathbf{B}^{-1}
\end{gathered}
$$

where $\Delta \mathbf{B}=-\mathbf{B}^{-1} \mathbf{A}\left(\mathbf{I}+\Delta \mathbf{B}^{\text {diag }} \mathbf{A}^{\prime} \mathbf{B}^{-1} \mathbf{A}\right)^{-1} \Delta \mathbf{B}^{\text {diag }} \mathbf{A}^{\prime} \mathbf{B}^{-1} . \quad$ As [2] demonstrate, the equivalent of (11) for a multi-element topology change is:

$$
\mathbf{F}^{\text {new }}=\mathbf{F}^{\text {old }}-\left[\Delta \mathbf{B}^{\text {diag }} \boldsymbol{\delta}^{\text {old }}-\left(\mathbf{B}^{\text {diag }}+\Delta \mathbf{B}^{\text {diag }}\right) \mathbf{A} ' \mathbf{R} \boldsymbol{\Gamma}\right] .
$$


Thus, the effects of a $n$-element topology change on the PTDF matrix can be broken down into a linear combination of $n$ single-element topology changes. Equations (8) and (12) can essentially be used interchangeably.

\section{CONGestion And Reliability Metrics}

The example of the Wheatstone network in Section III suggests two distinctions between reliability and congestion. The first is that reliability is a metric relating to contingencies, while congestion represents a constrained system during normal operations. The second is that congestion may increase the cost of serving the load, while a lack of reliability in the system implies that the desired level of demand cannot be met. Note that our definitions of reliability and congestion are from the point of view of the customer. The variables of interest are thus unserved energy and total system cost.

For concreteness, we will define a network topology $\Omega$ as a triple $\Omega=\left\{\mathbf{B}, \mathbf{A}, \mathbf{F}^{\max }\right\}$, where $\mathbf{B}$ is the susceptance matrix, $\mathbf{A}$ is the node-line adjacency matrix, and $\mathbf{F}^{\max }$ is the vector of capacity limits for the transmission lines. Unless stated otherwise, we will be comparing different network topologies under the following set of assumptions:

1. Demand remains constant following a change in network topology.

2. Demand is price-inelastic

3. The system operator behaves as if all customers have an equal value for reliability. Thus, unserved energy at a given node is inconsistent with excess generation capacity at that node. ${ }^{3}$

\section{A. Congestion Metrics}

A single line $l$ becomes congested when $F_{l}=F_{l}^{\max }$. From (8), we can see that a single-element topology change affecting line $k \neq l$ will cause congestion if $F_{l}^{\text {new }} \geq F_{l}^{\max }$, or [2], [3]:

$$
\begin{aligned}
& F_{l}^{\text {old }}+b_{k}^{-1} \mathbf{A}_{l}^{\prime} \mathbf{B}^{-1} \mathbf{A}_{k} B_{l} \delta_{k}^{\text {old }} \geq F_{l}^{\max } \\
& \quad \Rightarrow \Delta B_{k}^{-1} \geq \frac{\mathbf{A}_{l}^{\prime} \mathbf{B}^{-1} \mathbf{A}_{k} B_{l} \delta_{k}^{\text {old }}}{F_{l}^{\text {max }}-F_{l}^{\text {old }}}-\mathbf{A}_{k}{ }^{\prime} \mathbf{B}^{-1} \mathbf{A}_{k} .
\end{aligned}
$$

The congestion cost $C C$ of a change to the network topology is measured by taking the difference in the total cost of serving the load before and after the topological change:

$$
C C=\sum_{i} C_{i}\left(P_{G i, o p t}^{\Omega_{a}}\right)-\sum_{i} C_{i}\left(P_{G i, o p t}^{\Omega_{b}}\right),
$$

\footnotetext{
3 Another implication is that without some cost-based or value-based decision criteria, it may not always be clear which customer or customers should be blacked out in the event of a physical shortage of network transmission resources. We abstract from this decision problem and focus on the end state of the network; i.e., the sufficient system conditions for a customer to be blacked out.
}

where $\Omega_{a}$ and $\Omega_{b}$ represent two distinct network topologies, and $P_{G i, o p t}^{\Omega_{a}}, P_{G i, o p t}^{\Omega_{b}}$ represent the total-cost-minimizing output of the $i$ th generating unit with respect to network topologies $\Omega_{a}$ and $\Omega_{b}$.

\section{B. Reliability Metrics}

In this paper, we measure reliability using an unserved energy metric. ${ }^{4}$ Parameterizing the uncertainty surrounding a contingency on one of the transmission lines transforms this metric into the unserved energy expectation. Parameterizing customer demands using either a value of lost load (VOLL) or an explicit demand curve further transforms this metric into the expected value of unserved energy, as in [23].

A reliable system (with respect to a particular topological configuration) minimizes the need to physically ration consumption. We will define the system as reliable (with respect to a given topology $\Omega$ ) if $P_{L i}^{\Omega}=P_{d i}^{\Omega}$ for all $i, i=$ $\{1, \ldots, N B\}$, where $P_{d i}$ is the amount of power the customer at bus $i$ desires to consume, and $P_{L i}$ is the amount of power actually consumed.

We also define $\Delta P_{L i}=P_{L i}^{\Omega_{a}}-P_{L i}^{\Omega_{b}}$ to be the difference between consumption at the $i$ th bus with two different network topologies represented by $\Omega_{a}$ and $\Omega_{b}$. Thus, $\Delta \mathrm{P}_{L i}$ represents the (relative) reliability benefit of topology $\Omega_{a}$ over topology $\Omega_{b}$. We will also define a variable $\Delta \mathrm{P}_{G i}$ in a similar fashion to $\Delta \mathrm{P}_{L i}$.

Our focus in this paper is on situations where the initial network topology $\Omega_{a}$ represents a reliable system. For these systems, the assumption of a constant demand profile excludes the case $\Delta \mathrm{P}_{L i}>0$.

\section{SEPARABILITy AND DeComposition OF RELIABILITY AND CONGESTION}

In this section we use the decomposition method from (8) and (12) to derive some explicit topological conditions under which congestion and reliability will be independent. Sketches of proofs are provided here; complete and detailed proofs can be found in [2].

For a given net demand bus, with $P_{G i} \neq 0$, reliability decreases following a topological network change if the available transfer capacity (ATC) into bus $i$ declines. We use the (linear) ATC from all buses $s$ to bus $i$, defined as in [26], [27], [2]:

$$
A T C_{i}=\max _{s} \min _{m}\left\{T_{m}^{s}\right\}
$$

where $T_{m}^{s}=\left(F_{m}^{\max }-F_{m}\right) / \rho_{m, i}$.

Suppose that (15) is satisfied by line $l$, both before and after the topology change. Then the change in ATC is given

\footnotetext{
${ }^{4}$ Other reliability metrics include the $N-k$ criterion and the loss of load probability [24], [25].
} 
by:

$$
\Delta A T C=\frac{F_{l}^{\max }-\left(F_{l}^{\Omega_{a}}+\Delta F_{l}\right)}{\rho_{l, i}+\Delta \rho_{l, i}}-\frac{F_{l}^{\max }-F_{l}^{\Omega_{a}}}{\rho_{l, i}} .
$$

Note that $\triangle A T C<0$ if:

$$
\frac{\Delta F_{l}}{F_{l}^{\max }-F_{l}^{\Omega_{a}}}<\frac{\Delta \rho_{l, i}}{\rho_{l, i}}
$$

that is, if a network topology change causes a proportionally larger change in the distribution factor of the limiting line connected to bus $i$ than in the flow across the limiting line connected to bus $i$. This leads to the following results:

Result 1: Suppose that bus $i$ represents a net demander. Assume that $\Omega_{a}$ represents a reliable system, and that the change in topology from $\Omega_{a}$ to $\Omega_{b}$ represents a single-element topology change. Then reliability at bus $i$ decreases following the network topology change if $\Delta B_{k}^{-1}>\frac{\mathbf{A}_{l}^{\prime} \mathbf{B}^{-\mathbf{1}} \mathbf{A}_{k} B_{l} \delta_{k}^{\Omega_{a}}}{\left(F_{l}^{\max }-F_{l}^{\Omega_{a}}\right)} \frac{\rho_{l, i}}{\Delta \rho_{l, i}}-\mathbf{A}_{k}{ }^{\prime} \mathbf{B}^{-\mathbf{1}} \mathbf{A}_{k}$.

Proof of Result 1: The assumption that bus $i$ is a net demander is equivalent to assuming that (17) is satisfied, because a net demander will see a decrease in reliability if $A T C^{\Omega_{a}}>A T C^{\Omega_{b}}$. Inserting (6) into (17) yields:

$$
\frac{\mathbf{A}_{l}^{\prime} \mathbf{B}^{-\mathbf{1}} \mathbf{A}_{k} B_{l} \delta_{k}^{\text {old }}}{\Delta B_{k}^{-1}+\mathbf{A}_{k}{ }^{\prime} \mathbf{B}^{-1} \mathbf{A}_{k}} \rho_{l, i}<\Delta \rho_{l, i}\left(F_{l}^{\max }-F_{l}^{\Omega_{a}}\right),
$$

and then solving for $\Delta B_{k}^{-1}$ yields the result.

Result 2: Under the DC power flow assumptions with linear ATC, for a single-element topology change affecting line $k$, we have the following:

$$
\begin{gathered}
\Delta \rho_{l, i}=\rho_{l, i} \Leftrightarrow \Delta B_{k}^{-1}=\frac{\mathbf{A}_{l}{ }^{\prime}\left(\Delta \mathbf{B}_{i}^{-1}+\mathbf{B}_{i}^{-1}\right)}{\rho_{l, i}-B_{l} \mathbf{A}_{l}{ }^{\prime} \Delta \mathbf{B}_{i}^{-1}} \\
\Delta \rho_{l, i}>\rho_{l, i} \Leftrightarrow \Delta B_{k}^{-1}<\frac{\mathbf{A}_{l}{ }^{\prime}\left(\Delta \mathbf{B}_{i}^{-1}+\mathbf{B}_{i}^{-1}\right)}{\rho_{l, i}-B_{l} \mathbf{A}_{l}{ }^{\prime} \Delta \mathbf{B}_{i}^{-1}} \\
\Delta \rho_{l, i}<\rho_{l, i} \Leftrightarrow \Delta B_{k}^{-1}>\frac{\mathbf{A}_{l}{ }^{\prime}\left(\Delta \mathbf{B}_{i}^{-1}+\mathbf{B}_{i}^{-1}\right)}{\rho_{l, i}-B_{l} \mathbf{A}_{l}{ }^{\prime} \Delta \mathbf{B}_{i}^{-1}} .
\end{gathered}
$$

Proof of Result 2: We will prove the result for case (i); the proofs for cases (ii) and (iii) are identical. From (11), we see that $\Delta \rho_{l, i}=B_{l} \mathbf{A}_{l}{ }^{\prime} \Delta \mathbf{B}_{i}^{-1}+\Delta B_{k} \mathbf{A}_{l}{ }^{\prime}\left(\Delta \mathbf{B}_{i}^{-1}+\mathbf{B}_{i}^{-1}\right)$, where $\Delta \mathbf{B}_{i}^{-1}$ and $\mathbf{B}_{i}^{-1}$ represent the $i$ th column of $\Delta \mathbf{B}^{-1}$ and $\mathbf{B}^{-1}$. Thus, for case (i) to hold, we must have:

$$
\begin{aligned}
& \rho_{l, i}=B_{l} \mathbf{A}_{l}{ }^{\prime} \Delta \mathbf{B}_{i}^{-1}+\Delta B_{k} \mathbf{A}_{l}{ }^{\prime}\left(\Delta \mathbf{B}_{i}^{-1}+\mathbf{B}_{i}^{-1}\right) \\
& \Leftrightarrow \Delta B_{k}^{-1}=\frac{\mathbf{A}_{l}{ }^{\prime}\left(\Delta \mathbf{B}_{i}^{-1}+\mathbf{B}_{i}^{-1}\right)}{\rho_{l, i}-B_{l} \mathbf{A}_{l}{ }^{\prime} \Delta \mathbf{B}_{i}^{-1}}
\end{aligned}
$$

Cases (ii) and (iii) can be verified using the same procedure as in (18).

Result 2 is particularly important; it illustrates that congestion and reliability can rarely be considered independent. The significance of Result 2 is that the condition for a decrease in reliability, as given in Result 1 , takes the same form as the condition for an increase in congestion, as given in (13). Result 2 shows conditions on the PDTF matrix under which one or both of Result 1 and (13) will hold. Case (i) in Result 2 demonstrates a necessary and sufficient topological condition under which congestion and reliability cannot be independent. Cases (ii) and (iii) demonstrate topological conditions under which congestion and reliability may be independent. Case (ii) shows a situation in which congestion is a sufficient condition for a degradation in reliability, but not a necessary condition. Case (iii) shows a situation in which a degradation in reliability is a sufficient condition for congestion, but not a necessary condition.

The next result complements case (iii). The result shows that any topological change that degrades reliability always causes congestion.

Result 3: Suppose that bus $i$ represents a steady-state net demander. Assume that $\Omega_{a}$ represents a reliable system, and that the change in topology from $\Omega_{a}$ to $\Omega_{b}$ represents a singleelement topology change. If reliability at bus $i$ is degraded by the change in network topology, and if (16) is satisfied, then the change in network topology results in congestion on at least one line connected to bus $i$.

Proof of Result 3: Under the assumption that reliability at bus $i$ is degraded, Result 1 tells us that $\Delta B_{k}^{-1}>\frac{\mathbf{A}_{l} \mathbf{B}^{-\mathbf{1}} \mathbf{A}_{k} B_{l} \delta_{k}^{\Omega_{a}}}{\left(F_{l}^{\max }-F_{l}^{\Omega_{a}}\right)} \frac{\rho_{l, i}}{\Delta \rho_{l, i}}-\mathbf{A}_{k}{ }^{\prime} \mathbf{B}^{-\mathbf{1}} \mathbf{A}_{k}$.

Since $\Delta A T C_{i}<0$, we also know that $\Delta \rho_{l, i}>0$ and thus $\rho_{l, i} / \Delta \rho_{l, i}>0 .^{5}$ We must also have:

$$
\begin{aligned}
\Delta B_{k}{ }^{-1}> & \frac{\mathbf{A}_{l}^{\prime} \mathbf{B}^{-\mathbf{1}} \mathbf{A}_{k} B_{l} \delta_{k}^{\Omega_{a}}}{\left(F_{l}^{\max }-F_{l}^{\Omega_{a}}\right)}-\mathbf{A}_{k}{ }^{\prime} \mathbf{B}^{-1} \mathbf{A}_{k} \\
& >\frac{\mathbf{A}_{l}^{\prime} \mathbf{B}^{-\mathbf{1}} \mathbf{A}_{k} B_{l} \delta_{k}^{\Omega_{a}}}{\left(F_{l}^{\max }-F_{l}^{\Omega_{a}}\right)} \frac{\rho_{l, i}}{\Delta \rho_{l, i}}-\mathbf{A}_{k}{ }^{\prime} \mathbf{B}^{-\mathbf{1}} \mathbf{A}_{k} .
\end{aligned}
$$

\footnotetext{
${ }^{5}$ Since bus $i$ is a net demander, the limiting line in the ATC calculation should have $\rho_{l, i}>0$.
} 
The inequality represents the condition for a network topology change to cause congestion, which establishes the result.

Results 1 through 3 outline general conditions under which reliability and congestion could be independent. Note that the first three results are only valid when bus $i$ is a net demander. By Assumptions 1 and 3, net generation at a give bus is incompatible with a degradation in reliability at that bus. Since demand is assumed to be completely price inelastic, even expensive sources of generation can prevent demand from being unserved.

Results 2 and 3, taken together, demonstrate that case (ii) in Result 2 is the only situation when congestion and reliability may be independent. Case (i) from Result 2 illustrates a situation in which a single-element topological change will both cause congestion and decrease reliability. Result 3 demonstrates that a decrease in reliability is a sufficient condition for there to be an increase in congestion, but not a necessary condition. Congestion can occur without adverse effects to reliability.

Braess' Paradox in power systems is not uniquely associated with the Wheatstone network [19]; not every Wheatstone network will present a tradeoff between congestion and reliability. The last result demonstrates that the presence of an embedded Wheatstone network is (with one exception) a necessary condition for a tradeoff to exist between congestion and reliability.

Result 4: Suppose that bus $i$ represents a steady-state net demander. Assume that $\Omega_{a}$ represents a reliable system. Suppose that a single-element topology change from $\Omega_{a}$ to $\Omega_{b}$ causes congestion. A single-element topology change representing a network addition (so that $\Delta B_{k}>0$ ), can also provide a reliability benefit only if the network (under topologies $\Omega_{a}$ and $\Omega_{b}$ ) contains an embedded Wheatstone bridge. A single-element topology change representing a network outage (so that $\Delta B_{k}<0$ ) can provide a reliability benefit in a network without an embedded Wheatstone bridge if $-\Delta B_{k} \geq \frac{1}{2} \sum_{j \in \Omega_{a}} B_{j}$.

Proof of Result 4: We prove the result here for the special case of a series-parallel network with one source and one sink. Our intention is to give an idea of the general proof, which can be found in [2]. In such a series-parallel network network, the distribution factors are given by:

$$
\rho_{l, i}=\frac{B_{l}}{\sum_{j \in \Omega_{a}} B_{j}}
$$

Thus, a single-element topology change $\Delta B_{k}$ will cause congestion if $\frac{\Delta B_{k}}{\sum_{j} B_{j}}>\frac{F_{k}^{\max }}{P_{L i}}$. Note that a change in network topology will cause congestion but improve reliability if $\rho_{l, i} / \Delta \rho_{l, i} \leq 1$.

If $\rho_{l, i} / \Delta \rho_{l, i} \leq 1$, then we must have:

$$
\begin{aligned}
& \frac{B_{l}}{\sum_{j \in \Omega_{a}} B_{j}} \leq \frac{B_{l}}{\sum_{j \in \Omega_{a}} B_{j}+\Delta B_{k}}-\frac{B_{l}}{\sum_{j \in \Omega_{a}} B_{j}} \\
& \Leftrightarrow \frac{1}{2} \sum_{j \in \Omega_{a}} B_{j} \geq \sum_{j \in \Omega_{a}} B_{j}+\Delta B_{k} \\
& \Leftrightarrow-\Delta B_{k} \geq \frac{1}{2} \sum_{j \in \Omega_{a}} B_{j} .
\end{aligned}
$$

Since $B_{j} \geq 0$ for all $j$ in a series-parallel network, (21) can only hold if $\Delta B_{k}<0$; that is, if the topology change represents an outage on line $k$ in the transmission system.

We note that $-\Delta B_{k} \geq \frac{1}{2} \sum_{j \in \Omega_{a}} B_{j}$ should not happen very often in actual systems (even series-parallel systems), since it would require $B_{k}<0$ for most $k$. Result 4 demonstrates that congestion and reliability cannot represent tradeoffs (as in the four-bus example of Section III) unless the network contains an embedded Wheatstone bridge. Thus, broader conclusions can be drawn from the otherwise stylized network discussed in Section III.

\section{ILLUSTRATION ON THE IEEE 118-Bus NETWORK}

Section VI demonstrated that there are good theoretical reasons to think of congestion and reliability as being highly interrelated. This section links the theory in [2] with the simulations in [1], which empirically examines the relationship between congestion and reliability in four Wheatstone sub-networks embedded in the IEEE 118-bus network, as shown in Figure 4. 


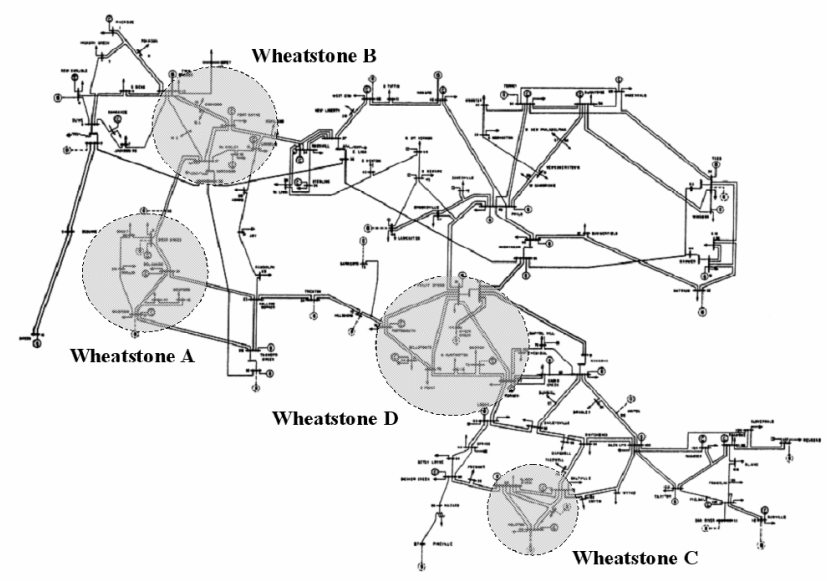

Figure 4: Four embedded Wheatstone networks in the IEEE 118-bus network.

The simulations in [1] define similar congestion and reliability metrics as in [2]. Congestion is defined using (14), while the reliability effect of the Wheatstone bridge is defined as:

$$
E V U E_{i}=u \times\left(\operatorname{VOLL} \times\left(P_{L i}^{\Omega_{a}}\right)-\operatorname{VOLL} \times\left(P_{L i}^{\Omega_{b}}\right)\right),
$$

where $u$ is the probability of a line outage on one of the Wheatstone boundary links. The net benefit of each Wheatstone bridge is defined as the reliability benefit minus the congestion cost.

For each Wheatstone sub-network in Figure 4, the simulation procedure in [1] solves the following optimization problem:

$$
\begin{aligned}
& \min _{P_{L i}, P_{G i}} \sum_{i}\left(C_{i}\left(P_{G i}\right)+V O L L_{i} \times U E_{i}\right) \\
& \text { s.t. } \\
& \sum_{j} F_{i j}^{\text {transfer }}=P_{G i}-P_{L i} \quad \forall i \\
& \mathbf{g}(\mathbf{x})=\mathbf{0} \\
& \mathbf{h}(\mathbf{x}) \leq \mathbf{0}
\end{aligned}
$$

where $\mathbf{g}$ and $\mathbf{h}$ represent the equality and inequality constraints in the system. The simulations in [1] consider a demand to transfer power across each Wheatstone subnetwork between 0 and $500 \mathrm{MW}$, and values for $u$ between $10^{-1}$ and $10^{-7}$.

We discuss here the simulation results for Wheatstone subnetworks C and D. As shown in [1], the results from Wheatstone sub-networks A and B are reasonably close to the results from Wheatstone sub-network $\mathrm{C}$.

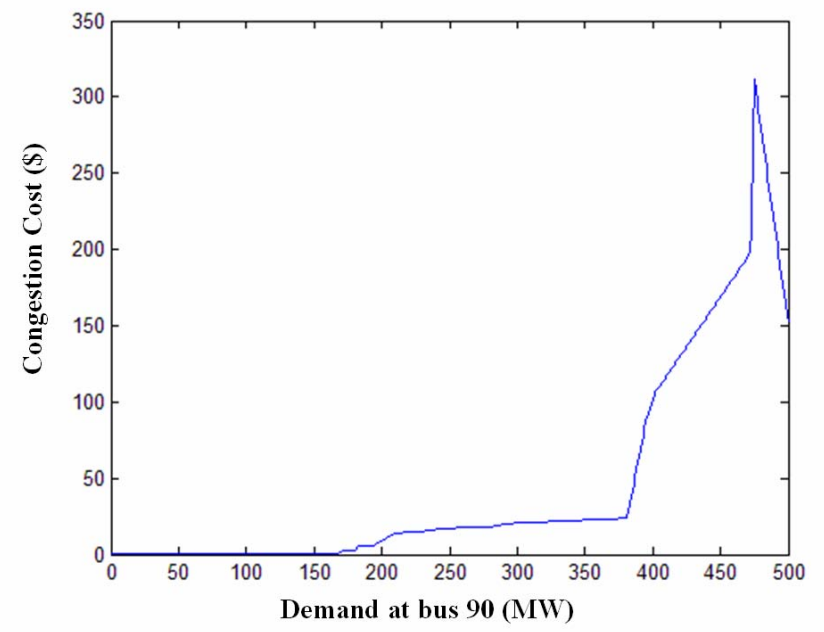

Figure 5: Congestion cost associated with Wheatstone sub-network C.

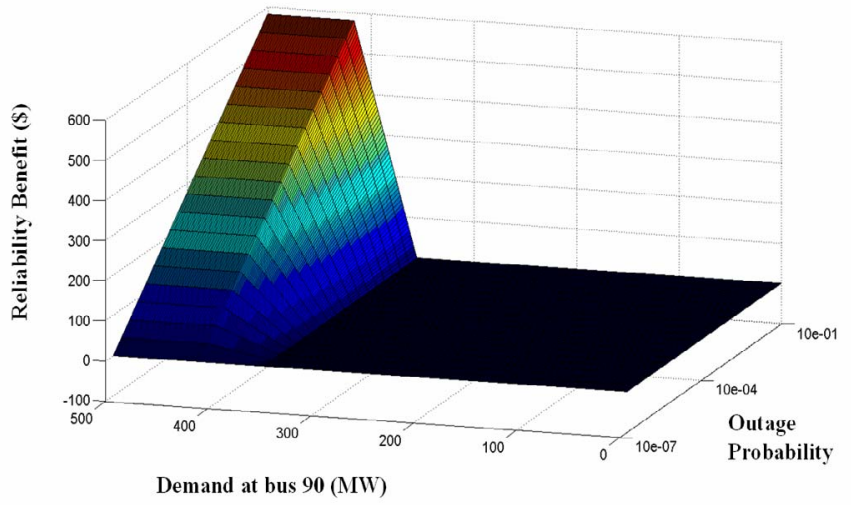

Figure 6: Monetized reliability benefit associated with Wheatstone sub-network C.

The congestion cost associated with the bridge in Wheatstone sub-network $\mathrm{C}$ is shown in Figure 5, while the (monetized) reliability benefit is shown in Figure 6. Just as with the four-bus Wheatstone network in Section III, the bridge causes congestion but also provides an (expected) reliability benefit. Figure 7 shows the congestion cost for the bridge in Wheatstone sub-network D, while Figure 8 shows the monetized reliability benefit.

Two differences between Wheatstone sub-networks $\mathrm{C}$ and $\mathrm{D}$ are apparent. The first is that the congestion cost of the bridge in Wheatstone $\mathrm{D}$ follows much more of a roller-coaster pattern than the bridge in Wheatstone $\mathrm{C}$, while the reliability benefit of the bridge in Wheatstone D behaves similarly to Wheatstone $\mathrm{C}$. The monetized reliability benefit is increasing both in the level of demand and in the probability of an outage. The magnitude of the congestion cost is also generally greater than the reliability benefit, so the pattern of congestion cost dominates the net benefit calculation. The other difference is that in Wheatstone $\mathrm{D}$, the congestion cost is usually negative. Thus, the bridge in Wheatstone $\mathrm{D}$ relieves congestion, rather than causing congestion. Reliability and congestion are complements in Wheatstone sub-network D. 


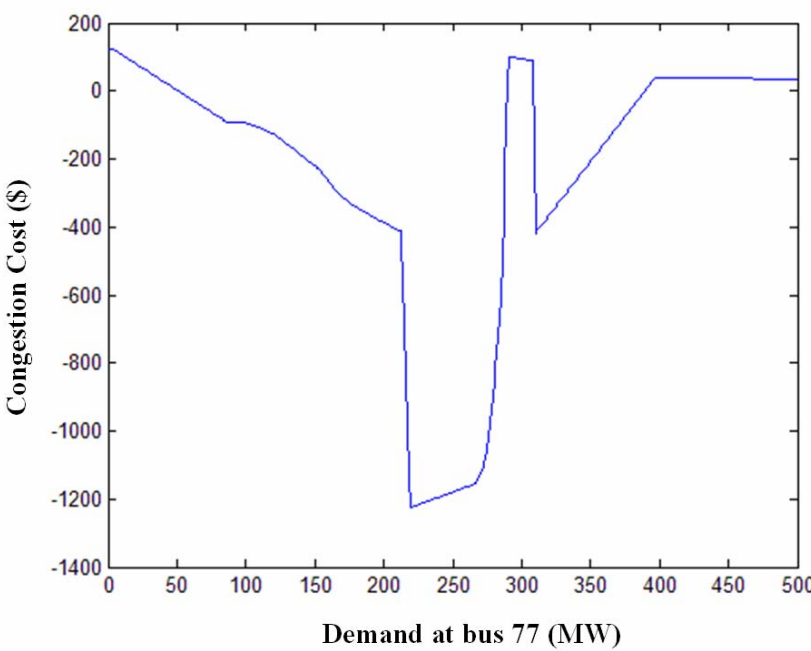

Figure 7: Congestion cost associated with Wheatstone sub-network D.

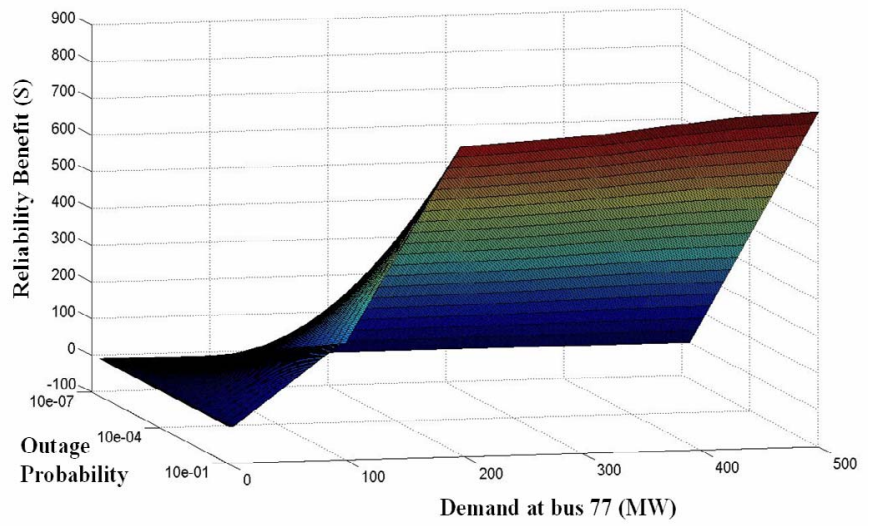

Figure 8: Monetized reliability benefit associated with Wheatstone sub-network D.

The behavior of the congestion cost function in Figure 7 is closely related to the location of Wheatstone sub-network D in the IEEE 118-bus network. This particular sub-network is located in an area of the grid in close proximity to a number of inexpensive sources of generation. If the cost of these nearby generators is increased, then the complementarity between reliability and congestion in Wheatstone sub-network $\mathrm{D}$ disappears; the new relationship is more like the tradeoff faced in Wheatstone networks A, B, and C.

\section{DISCUSSION AND CONCLUSIONS}

Reliability and congestion are rarely independent. Network investments made with reliability in mind can have effects on congestion (positive or negative), and vice versa. Further, these externalities are highly variable with the network topology and the level of demand, and may not be captured in locational prices [11], [3]. One planning solution is thus to construct enough network capacity so that demand, even peak demand, looks small by comparison. This, however, would be costly, and would ignore the effects of future planned investments [28]. The economics of the merchant transmission model are certainly problematic, as demonstrated in [11], for example. The discussion here suggests a more fundamental problem with the merchant model and its variations such as participant funding. We have shown that attempting to separate transmission investments into those that just reduce congestion and those that just enhance reliability is a fruitless venture. Transmission investment is a systems problem and should be treated as such.

The cost of congestion is straightforward to define. The interdependence of congestion and reliability suggests that reliability should be defined in terms of these costs and benefits. Particularly with the transition to markets in the new electric power industry, and with the increasing difficulty of siting and building new transmission lines, the value of reliability becomes a more important concept than physical reliability itself.

The four-bus Wheatstone network in Section III provides a good framework for illustrating concepts, but there are important differences between the test network and actual highly interconnected networks. Congestion and reliability do represent tradeoffs in three of the four Wheatstone networks embedded in the IEEE 118-bus network. In the fourth sub-network, congestion and reliability are complements, but certainly not independent. There are important insights to be gained from the four-bus test network.

The Wheatstone network provides a good framework for evaluating the value of flow-control devices such as FACTS. Ideally, the Wheatstone network would be operated in such a way that the bridge circuit would be opened during normal operations, but closed during contingencies in order to provide additional paths between the source and sink buses. Our future work in this area is closely related to these questions of valuing FACTS and assessing the true expected value of reliability.

The analysis in this paper provides a framework for assessing whether a new line will have congestion or reliability impacts (or both). Which effect dominates, and whether the impact is large or small, is ultimately an empirical matter.

\section{ACKNOWLEDGMENTS}

The authors would like to thank William Hogan, Paul Joskow, Paul Hines, Jose Moura, Jeffrey Roark, Dmitri Perekhodtsev, and Sarosh Talukdar for helpful comments and discussions.

\section{REFERENCES}

[1] Blumsack, S., L. B. Lave and M. Ilić, 2006. "A Quantitative Analysis of Congestion and Reliability in Electric Power Networks," CEIC working paper 06-09, available online at http://wpweb2.tepper.cmu.edu/ceic/papers/ceic-06-09.asp.

[2] Blumsack, S., M. Ilić and L. B. Lave, 2006. "Decomposing Congestion and Reliability," CEIC working paper 06-11, available online at http://wpweb2.tepper.cmu.edu/ceic/papers/ceic-06-11.asp.

[3] Blumsack, S., 2006. "Network Topologies and Transmission Investment Under Electric Industry Restructuring," Ph.D. Dissertation, Department of Engineering and Public Policy, Carnegie Mellon University. Available online 
http://wpweb2.tepper.cmu.edu/ceic/pdfs_other/Seth_Blumsack_PhD_Thesi s_2006.pdf

[4] NERC Reliability Standard IRO-006-01, available online at ftp://www.nerc.com/pub/sys/all_updl/standards/rs/IRO-006-1.pdf.

[5] P. Hines, J. Apt, H. Liao, and S. Talukdar, "The Frequency of Large Blackouts in the United States Electrical Transmission System: An Empirical Study," presented at the $2^{\text {nd }}$ Carnegie Mellon Conference on Electric Power, January 2006, available at http://www.ece.cmu.edu/ electriconf/hines_blackout_frequencies_final.pdf

[6] Hogan, W., 1992. "Contract Networks for Electric Power Transmission," Journal of Regulatory Economics 4: pp. 211 - 242.

[7] Bushnell, J. and S. Stoft, 1996. "Electric Grid Investment Under a Contract Network Regime," Journal of Regulatory Economics 10, pp. 61 -79 .

[8] Chao, H. and S. Peck, 1996. "A Market Mechanism for Electric Power Transmission," Journal of Regulatory Economics 10, pp. 25 - 59.

[9] Oren, S., 1997. "Economic Inefficiency of Passive Transmission Rights in Congested Electricity Systems with Competitive Generation," Energy Journal 18, pp. 63 - 83.

[10] Joskow, P. and J. Tirole, 2000. "Transmission Rights and Market Power on Electric Power Networks," RAND Journal of Economics 31:3, pp. 450 $-487$.

[11] Joskow, P. and J. Tirole, 2005. "Merchant Transmission Investment," Journal of Industrial Economics 53:2, pp. 233 - 264.

[12] Joskow, P., 2005. "Patterns of Transmission Investment," available at econ-www.mit.edu/faculty/index.htm?prof_id=pjoskow\&type=paper.

[13] DOE National Transmission Congestion Study, available online at http://www.oe.energy.gov/energy_policy/epa_sec1221.htm\#National_Elect ric_Transmission_Congestion_Study.

[14] Hebert, C., 2004. "Profit Without Costs," Public Utilities Fortnightly, $142: 8$, pp. $40-48$.

[15] Roark, J., 2006. "Redefining Merchant Transmission and Making it Happen," presented at the $2^{\text {nd }}$ Carnegie Mellon Conference on Electric Power, January 2006, available at http://www.ece.cmu.edu/ electriconf/Roark_ReDefining\%20Merchant $\% 2$ 0Transmission.pdf.

[16] Joskow, P., 2005. "The Difficult Transition to Competitive Electricity Markets in the U.S.," in Electricity Deregulation: Choices and Challenges, J. Griffin and S. Puller, eds., University of Chicago Press, Chicago.

[17] Braess, D., 1968. "Über ein Paradoxon aus der Verkehrsplanung," Unternehmensforschung 12, pp. 258 - 268.

[18] Arnott, R. and K. Small, 1994. "The Economics of Traffic Congestion," American Scientist 82, pp. 446 - 455.

[19] Blumsack, S. and M. Ilić, 2006. "The Braess Paradox in Electric Power Networks," working paper, available online at http://www.andrew.cmu.edu/user/sblumsac/braess_paradox.pdf.

[20] Ejebe, G. and B. Wollenberg, 1979. "Automatic Contingency Selection," IEEE Transactions on Power Apparatus and Systems PAS-98, pp. 97 109.

[21] Irisarri, G., D. Levner, and A. Sasson, 1979. "Automatic Contingency Selection for On-Line Security Analysis - Real-Time Tests," IEEE Transactions on Power Apparatus and Systems PAS-98, pp. 1552 - 1559.

[22] Baldick, R., 2003. "Variation of Distribution Factors with Loading," IEEE Transactions on Power Systems 18:4, pp. 1316 - 1323.

[23] Zerriffi, H., H. Dowlatabadi, and A. Farrell, 2006. "Incorporating Stress in Electric Power Systems Reliability Models," Energy Policy, forthcoming.

[24] Coxe, R. and M. Ilić, 1998. "System Planning Under Competition," in Power Systems Restructuring: Engineering and Economics, M. Ilić, F. Galiana, L. Fink, eds., Kluwer Academic Publishing, Boston MA.

[25] Choi, J., T. Mount and R. Thomas, 2006. "Transmission System Expansion Plans in View Point of Deterministic, Probabilistic and Security Reliability Criteria," Proceedings of the $39^{\text {th }}$ Hawaii International Conference on System Sciences.

[26] Grijalva, S., P. Sauer, and J. Weber, 2003. "Enhancement of Linear ATC Calculations by the Incorporation of Reactive Power Flows," IEEE Transactions on Power Systems 18:2, pp. 619 - 624.

[27] Ilić, M., F. Galiana, L. Fink, A. Bose, P. Mallet, and H. Othman, 1998. "Transmission Capacity in Power Networks," International Journal on Electrical Power and Energy Systems 20:2, pp. 99 - 110.
[28] Sauma, E. and S. Oren, 2006. "Proactive Planning and Valuation of Transmission Investments in Restructured Electricity Markets," Journal of Regulatory Economics 30:3, pp. 261 - 290. 